\title{
Desarrollo de competencias científicas en las primeras edades: el Explora Conicyt de Chile
}

\author{
Marta Elsa Quiroga-Lobos \\ Escuela de Pedagogía \\ Pontificia Universidad Católica de \\ Valparaíso, Chile \\ quirogamarta846@gmail.com
}

\section{Emanuel Arredondo-González}

Magíster en Educación

Pontificia Universidad Católica de

Valparaíso, Chile

emanuel.arredondo@ucv.cl

\section{Cristian Merino-Rubilar}

Instituto de Ouímica

Pontificia Universidad Católica de

Valparaíso, Chile

cristian.merino@ucv.cl

\section{Resumen}

Este trabajo presenta los resultados de la implementación en jardines infantiles de la iniciativa Tus Competencias Cientificas en Ciencias (TCC) Explora Conicyt y analiza la influencia de las relaciones colaborativas entre directores, educadoras de párvulos y asistentes de párvulos en el desarrollo de competencias científicas de alumnos entre 4 y 6 años.

La metodología fue de carácter mixto. Se realizaron 37 observaciones de aula que se analizaron a través del método comparativo constante. Surgieron diez categorías de colaboración, utilizadas en la construcción de un cuestionario para los tres actores educativos. El cuestionario se aplicó en 97 instituciones educativas municipales y particulares de las regiones de Valparaíso, La Araucanía, Los Lagos, Los Ríos y Metropolitana.

Los resultados muestran una correlación positiva entre colaboración de los agentes educativos y los aprendizajes científicos de los estudiantes.

\section{Palabras clave}

Educación primera infancia, enseñanza de ciencias básicas, formación de profesores, gestión escolar, Chile. (Fuente: Tesauro de la Unesco).

Recepción: 2013-08-06 / Envío a pares: 2014-03-16 / Aceptación por pares: 2014-07-10/ Aprobación: 2014-07-25 DOI: 10.5294/edu.2014.17.2.2

Para citar este artículo / To reference this article / Para citar este artigo

Quiroga-Lobos, M. , Arredondo-González, E., Cafena, D. y Merino-Rubilar, C. (2014). Desarrollo de competencias científicas en las primeras edades: el Explora Conicyt de Chile. Educ. Educ. 17 (2), 237-253. Doi 10.5294/edu.2014.17.2.2 


\title{
Development of Scientific Expertise in Early Childhood: Explora Conicyt in Chile
}

\begin{abstract}
The paper presents the results of the implementation of a preschool school initiative in Chile known as Your Scientific Skills in Science (TCC) Explora Conicyt and analyzes the influence of cooperative relationships between directors, preschool teachers and preschool teaching assistants on the development of scientific skills in students between four and six years of age.

A mixed methodology was applied. Thirty-seven classroom observations were conducted and analyzed using the constant comparative method. Ten categories of cooperation emerged and were used to construct a questionnaire for these three educational players. The questionnaire was applied at ninety-seven municipal and private schools in the regions of Valparaíso, La Araucanía, Los Lagos, Rivers and Metropolitana. The results show a positive correlation between cooperation among educators and scientific learning on the part of students.
\end{abstract}

Key words

Early childhood education, basic science education, teacher training, school management, Chile. (Source: Unesco Thesaurus). 


\section{Desenvolvimento de competências científicas nas primeiras idades: o Explora Conicyt do Chile}

\section{Resumo}

Este trabalho apresenta os resultados da implantação em jardins infantis da iniciativa "Tus Competencias Cientificas en Ciencias" (TCC) Explora Conicyt e analisa a influência das relações colaborativas entre diretores, educadoras de crianças e assistentes de crianças no desenvolvimento de competências científicas de alunos entre 4 e 6 anos.

A metodologia foi de caráter misto. Realizaram-se 37 observações de sala de aula que foram analisadas por meio do método comparativo constante. Surgiram 10 categorias de colaboração, utilizadas na construção de um questionário para os três atores educativos. O questionário foi aplicado em 97 instituições educativas municipais e particulares das regiões de Valparaíso, La Araucanía, Los Lagos, Los Ríos e Metropolitana.

Os resultados mostram uma correlação positiva entre colaboração dos agentes educativos e as aprendizagens cientificas dos estudantes.

\section{Palavras chave}

Educação primeira infância, ensino de ciências básicas, formação de professores, gestão escolar, Chile. (Fonte: Tesauro da Unesco). 


\section{Introducción}

Aprender ciencias hoy se ha convertido en la piedra angular de la educación del siglo XXI, y se visualiza como el motor para la formación de ciudadanos comprometidos y cruciales para la democracia, dado que puede mejorar de forma sustancial la vida de las personas, generar movilidad social y conferir el poder para ser actores y no espectadores de los productos de la ciencia (Michaels, Shouse y Schweingruber, 2007; González-Weil, Martínez, Martínez, Cuevas y Muñoz, 2009). El aumento de la importancia de las ciencias en el mundo moderno ha traído aún más atención sobre la enseñanza de las ciencias en la educación básica y media, con el objetivo de que los estudiantes sean capaces de usar el conocimiento científico, identificar problemas y sacar conclusiones basadas en evidencia, a fin de comprender y ayudar a tomar decisiones sobre el mundo natural y los cambios producidos en él a causa de la actividad humana.

De acuerdo con este planteamiento inicial, en los últimos diez años los diferentes gobiernos de Chile, a través de su agenda política, han instalado como prioridad la formación en ciencias de manera temprana y oportuna desde las primeras edades, mediante diferentes iniciativas formales e informales. Un ejemplo de ello es el Programa Explora y la iniciativa Tus Competencias en Ciencias (TCC), que consiste en un taller extracurricular que se implementa en establecimientos educativos y que tiene por finalidad desarrollar competencias para la valoración de la ciencia y la tecnología en los estudiantes.

No obstante lo anterior, creemos que la promoción y el desarrollo de competencias en ciencias desde las primeras edades no es algo que emerge de forma espontánea producto de la interacción de los niños con unos materiales para la resolución de un problema y unas orientaciones proporcionadas por las educadoras de párvulos. Desde nuestra experiencia, como parte del equipo que formó a las educadoras que implementaron el programa TCC en diferentes establecimientos del país, pudimos evidenciar que este comenzó a generar un gran interés que dio como resultado una relación colaborativa entre directores, educadoras y asistentes de párvulos que participaron del programa durante veinte semanas. Por tanto, ¿las relaciones entre estos actores educativos podrían ser potencialmente un factor preponderante en la promoción y el desarrollo de competencias científicas en estudiantes entre 4 y 6 años?

\section{Antecedentes teóricos sobre colaboración entre actores educativos para la promoción de competencias en ciencias desde las primeras edades}

Las instituciones escolares han sido definidas como complejas (Tyler, 1996); la multiplicidad de relaciones que se establecen en su interior requieren ser organizadas y conducidas, para que todas ellas coincidan en generar condiciones apropiadas para el aprendizaje de los estudiantes. La academia ha desarrollado distintas aproximaciones para explicar la necesidad de colaboración entre los docentes, las que se asocian a liderazgo distribuido' que, según Bennett, Wise, Woods y Harvey (2003), se caracteriza en primer lugar por ser una propiedad emergente de un grupo o red de individuos que interactúan entre sí y que produce un resultado que es más que la suma de las acciones individuales (Gronn, 2002);y en segundo lugar, presenta apertura en los límites del liderazgo, es decir, si bien no determina cuántos tienen que participar, tampoco marca una frontera determinada en donde el liderazgo tiene que dejar de ser compartido. Finalmente, en tercer lugar, enfatiza que existe una variedad de experiencia distribuida entre muchos y que, por tanto, se pueden encontrar diferentes capacidades y perspectivas en individuos

1 Aunque el concepto de liderazgo distribuido se remonta a la psicología social de los años cincuenta (Dinham, Aubusson y Brady, 2008), es a partir de los años ochenta que la investigación del liderazgo escolar comienza a abandonar el foco centrado en el director como líder fundamental para enfatizar la responsabilidad colectiva y la actividad colegiada de grupos de profesores, introduciéndose la noción de liderazgo distribuido (Ávila, 2008). 
diseminados por toda la organización, los que, trabajando juntos, forjan una dinámica concertada que suma más que las contribuciones individuales. Las iniciativas, si bien pueden ser emprendidas por aquellos con habilidades relevantes en un contexto particular, luego son adoptadas, adaptadas y mejoradas por los otros, dentro de una cultura de confianza y apoyo mutuo. El liderazgo, de esta manera, se distribuye tanto entre líderes posicionales como informales (Spillane, Halverson y Diamond, 2001) y se produce en una red de relaciones (Ogawa y Bossert, 1995), donde la clave no son los actos ni los actores, sino las interacciones (Bolden, 2007).

El liderazgo distribuido, como una propiedad de toda la organización (Ávila, 2008), se entronca con el concepto de liderazgo como influencia planteado por Bush (2008), en que los liderazgos formales y de muchos otros individuos movilizan y guían a otros profesores en un proceso de cambio instruccional (Harris, 2002). Un modelo de liderazgo distribuido se enfoca en las interacciones simétricas más que en las acciones de aquellos en roles formales e informales de liderazgo (Harris y Spillane, 2008, p. 1). En este modelo "las interacciones entre líderes tienen una relación de reciprocidad en la que la solidaridad y el trabajo en equipo tienen prioridad" (Riveros-Barrera, 2012, p. 297), conformando comunidades de aprendizaje con fuertes lazos sociales y profesionales. Bryk, Camburn y Seashore (1999) han encontrado una relación dinámica entre la confianza y la creación de comunidades profesionales, la que se funda en las relaciones entre profesores que son apoyados por directores que mantienen un contacto permanente y tienen estilos inclusivos de liderazgo. De manera directa aumentan los sentimientos de pertenencia y propiedad sobre la organización y, por tanto, el compromiso y la moral de los equipos de trabajo, lo que se expresa en una mejor comunicación y mayor cooperación; de manera indirecta mejora el proceso de toma de decisiones y los resultados de los estudiantes (Harris y Townsend, 2007; Harris y Spillane, 2008; Southard, Muldoon, Porter y Hood,
1997). Una investigación realizada por GroupEducation (Arrowsmith, 2004) determinó que el ejercicio del liderazgo distribuido crea un cierto clima en la escuela que se refleja en logros, asistencia y "otras mediciones duras", aunque es claro que no se puede establecer una conexión directa con los resultados académicos, pero sí en aspectos propios del trabajo del equipo, tales como moral, creatividad y compromiso con los valores comunes.

La conformación de comunidades de práctica requiere la transferencia de funciones de liderazgo, lo que demanda la existencia de estructuras de conocimiento compartidas y de un clima que haga que los miembros sientan aceptable tener intercambios de roles de liderazgo fluidos. La confluencia de estos factores permite una suave transferencia del liderazgo de un miembro a otro y facilita el comportamiento adaptativo del equipo (Burke, Stagl, Salas, Pierce y Kendall, 2006, p. 123). Se requiere que los miembros se sientan cómodos en un ambiente de reflexión colectiva, y que estén dispuestos a dar y recibir opiniones (Conley, Fauske y Pounder, 2004), lo que significa aprender constantemente y en comunidad con otros.

Si estamos de acuerdo en las tres dimensiones que, para Woods, Bennett, Harvey y Wise (2003) caracterizan el liderazgo distribuido, es evidente que el ejercicio de este requiere de condiciones organizativas, estructurales y personales que permitan que el liderazgo sea ejercido por muchos en su práctica efectiva cotidiana

Cranston (2009) destaca, de un estudio realizado con directores en Canadá, "la gran cantidad de tiempo y esfuerzo que es necesario invertir para que los profesores coordinen su trabajo" (p. 248). La coordinación pedagógica de los docentes demanda el desarrollo de consensos que van más allá de la simple distribución de tareas, ya que implican consensuar expectativas, creencias, conocimientos y rutinas de cómo se hacen las cosas en un determinado momento en el aula. Es decir, en palabras de Har- 
greaves (2001), se hace necesario desarrollar capital social al interior de la institución, el que permitirá ampliar la seguridad y confianza en el equipo para compartir el capital intelectual². Un capital social alto entraña altos niveles de confianza y es un importante lubricante para la transferencia de conocimiento, del que depende la movilización del capital intelectual de una organización (p.492).

Para concluir, la calidad de las interacciones profesionales entre directores, educadoras y asistente de párvulos crea condiciones propicias para el intercambio de conocimientos, desarrollo de acciones colaborativas en el aula, estrategias de apoyo para la implementación del taller TCC y distribución de tareas.

\section{Las ciencias como parte de la cultura de la humanidad y el desarrollo de competencias científicas desde las primeras edades}

Las ciencias forman parte de la cultura de la humanidad y una de sus preocupaciones es comprender el mundo natural y los cambios que la actividad humana produce en función del conocimiento que genera. Para conseguir estos objetivos busca identificar preguntas relevantes, generar conceptos, modelos y teorías para dar respuesta a fenómenos del mundo y encontrar pruebas que las confirmen o den lugar a nuevas preguntas (Ouiroga, Cafena, Merino y Olivares, 2012).

Enseñar ciencias en educación parvularia permitiría a las educadoras ayudar a niños y niñas a apropiarse de esta cultura, a saber utilizarla y a ge-

2 El capital intelectual es el conocimiento organizado que puede ser utilizado, es decir, la suma del conocimiento de todos los miembros de la organización que crece por la creación de nuevo conocimiento y por la transferencia de conocimiento entre personas y situaciones. El capital social tiene un componente cultural, consistente en el nivel de confianza entre las personas y la generación de normas de reciprocidad y colaboración, y un componente estructural, que es la red en que las personas están ligadas por fuertes lazos. En un capital social rico existen altos niveles de confianza que se manifiestan en redes fuertes y relaciones de colaboración entre sus miembros y socios (Hargreaves, 2001). nerar el deseo de hacerla evolucionar. En el aprendizaje de la ciencia se entrelazan muchos factores: los intereses, la observación y la experimentación, las estrategias de razonamiento, la manera de organizar las ideas, la forma de comunicarlas y los valores. Además, el conocimiento y las destrezas que se adquieren son útiles para "transformar" a niños y niñas en ciudadanos mejor formados en un mundo que se hace cada vez más complejo y más interesante desde el punto de vista tecnológico.

A pesar de que existen diversas definiciones sobre lo que es una competencia científica (Rebollo, 2010), recurrimos a la definición propuesta por el proyecto PISA (2006), en donde esta se define como:

la capacidad de emplear los conocimientos científicos de un individuo y el uso de ese conocimiento para identificar problemas, adquirir nuevos conocimientos, explicar fenómenos cientificos y extraer conclusiones basadas en pruebas sobre cuestiones relacionadas con la ciencia. Asimismo, comporta la comprensión de los rasgos característicos de la ciencia, entendida como un método del conocimiento y la investigación humanas, la percepción del modo en que la ciencia y la tecnología conforman nuestro entorno material, intelectual y cultural, y la disposición a implicarse en asuntos relacionados con la ciencia y con las ideas de la ciencia como un ciudadano reflexivo (p. 9).

Esta definición reconoce que la competencia científica se compone de tres dimensiones: los conocimientos, las habilidades y las actitudes, identificando catorce subdimensiones en total (Rebollo, 2010).

Para desarrollar competencias científicas, la enseñanza de las ciencias debe plantearse como finalidad transformar el aula en un espacio de construcción del conocimiento científico escolar para la comprensión de los fenómenos y la participación activa del sujeto en un contexto sociocultural susceptible de ser transformado. Sin embargo, la actividad científica escolar tiene por finalidad central cons- 
truir modelos teóricos escolares que permitan comprender los fenómenos naturales, intervenir en ellos y establecer juicios de valor sobre el proceso y sus resultados (Izquierdo y Adúriz-Bravo, 2003), además de incorporar paulatinamente las ideas consensuadas en la comunidad científica. Es esencial mostrar la ciencia a los niños y las niñas como una actividad humana racional, pero de manera moderada.

Para el logro de este propósito el programa TCC se encuentra compuesto de treinta experiencias científicas que fueron diseñadas a partir de los siguientes principios: a) reconocer los conocimientos previos e intereses de los niños y las niñas; b) potenciar la experimentación a través de la manipulación de materiales; c) generar instancias de intercambio verbal entre compañeros; d) estimular la retroalimentación indagatoria sobre sus observaciones y que las actividades contribuyan al desarrollo de las competencias científicas.

\section{Formación inicial y desarrollo profesional de las educadoras de párvulos en ciencias}

La investigación sugiere que el conocimiento científico de las educadoras es predictivo respecto al aprendizaje en ciencias de sus estudiantes (LoucksHorsley, Love, Stiles, Mundry y Hewson, 2003, citado en Gropen, Clark-Chiarelli, Ehrlich y Thieu, 2011). Existen estudios que muestran que el conocimiento del profesorado y de las educadoras sobre ciencia influye poderosamente en la manera de interpretar y de actuar en la enseñanza. Por ejemplo, un estudio desarrollado por Gropen et al. (2011) demostró que un grupo de educadoras de párvulos participantes en un programa de desarrollo profesional en ciencias mostró fuertes cambios en sus conocimientos y prácticas de enseñanza, lo que implicó que sus estudiantes evidenciaran una tendencia a incrementar su comprensión sobre los principios físicos de la ciencia y el uso de sus habilidades de investigación científica.

En el caso de Chile, las carreras de Educación Parvularia carecen de formación específica en ciencias (García-Huidobro, 2006), por lo que se hace necesario implementar procesos de formación continua (Bowman et al., 2001; Dwyer, Chait y McKee, 2000; Espinosa, 2002; Helburn y Bergmann, 2002). A partir de esta constatación es que el proyecto TCC Educación Parvularia desarrolla un proceso de capacitación inicial e intermedia de 16 horas cronológicas, en el que se revisan las características centrales de las actividades por desarrollar con los párvulos, se entrega la carpeta metodológica y el kit de materiales. El acompañamiento entre las sesiones presenciales se realiza a través de una plataforma virtual. Adicionalmente, se efectúan encuentros presenciales de reflexión.

\section{Materiales y métodos}

De acuerdo con lo planteado en la introducción y en el marco teórico, la investigación se ha propuesto ampliar el conocimiento sobre cómo las relaciones de colaboración/coordinación entre los actores educativos (director, educadora y asistente de párvulos) son potencialmente un factor preponderante en la promoción y el desarrollo de competencias científicas en estudiantes entre 4 y 6 años, bajo el marco de la iniciativa Tus Competencias en Ciencias para Educación Parvularia, de Explora (Conicyt).

Con la finalidad de contestar esta pregunta se desarrolló una investigación mixta de carácter exploratorio y descriptivo (Hernández, Fernández y Baptista, 2010). La investigación se dividió en dos partes: inicialmente se realizaron 37 observaciones de aula, y de su análisis se identificaron factores que incidían en el nivel de desarrollo de competencias científicas de los estudiantes. Posteriormente estos factores fueron operacionalizados (Asún, 2006) en un cuestionario que fue aplicado a directores, educadoras y asistentes de párvulos, y cruzados con los resultados de los estudiantes.

\section{Participantes}

Para la realización del estudio se contó con los establecimientos educacionales $(\mathrm{N}=97)$ que participaron de la primera convocatoria de EXPLORA TCC- 
EP distribuidos en cuatro regiones del país: Valparaíso, La Araucanía, Los Lagos y Los Ríos. De estos solo se trabajó con los resultados de 72 instituciones, descartando del análisis a los establecimientos de los que no se contaba con la información de los cuatro actores educativos 3 . En la tabla 1 se presenta en detalle la distribución de los establecimientos.

Tabla 1. Muestra de establecimientos

\begin{tabular}{lcc}
\hline \multicolumn{1}{c}{ Región } & N & \% \\
\hline Valparaíso & 49 & 68,06 \\
Los Ríos & 13 & 18,06 \\
Los Lagos & 2 & 2,78 \\
La Araucanía & 8 & 11,11 \\
\hline Total & 72 & 100
\end{tabular}

\section{Instrumento}

El instrumento fue construido a partir de la bibliografía y de los resultados de 37 observaciones de aula, las cuales se sistematizaron y analizaron aplicando el método comparativo constante. Emergieron así categorías que responden a los elementos de mayor importancia para generar condiciones institucionales y de aula a fin de desarrollar las competencias de los párvulos. Estos elementos se presentan en la figura 1:

\section{Figura 1. Categorización emergente}

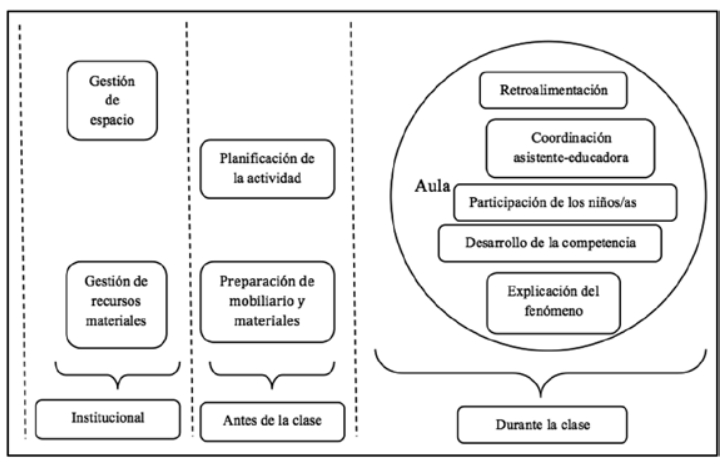

3 Director, educadora de párvulos, asistente de párvulos y estudiantes.
En la figura 1 se evidencia que la mayor parte de las categorías se encuentran asociadas al rol de mediación de la educadora y asistente de párvulos durante la implementación de la actividad, es por esto que resulta relevante observar cómo ellas colaborativamente generan un clima estimulante para el desarrollo de las competencias científicas de los párvulos, realizando algunas de las siguientes acciones: retroalimentación verbal y gestual a los niños y las niñas, realización de explicaciones de calidad sobre los fenómenos, estimulación de la participación y protagonismo de los niños y las niñas, interacción entre asistente y educadora con foco en los aprendizajes de estudiantes y focalización de su atención en el desarrollo de la competencia.

Las categorías evidenciadas en el "hacer la clase", es decir, durante la experiencia científica, se generan a partir de ciertas condiciones institucionales que las facilitan, como el planificar conjuntamente, lo que les permite construir un sentido compartido sobre los roles que deben desempeñar durante el desarrollo de la actividad y generar un escenario propicio para el desenvolvimiento de la misma. Desde esta perspectiva, el rol del director es clave ya que genera condiciones y gestiona los recursos institucionales, como por ejemplo, el tiempo de las docentes para planificar en forma conjunta, organizar espacios físicos y temporales para la ejecución de las actividades, implementar estrategias y reponer materiales e insumos para la realización de las experiencias científicas. Estas ideas se concretan en los ejes que dan origen a las preguntas formuladas en los cuestionarios que se aplican al universo de los participantes de este proyecto. Las dimensiones que son evaluadas son las que se presentan en la tabla 2.

En función de estas diez dimensiones descritas en la tabla 2 se construyó un cuestionario que fue aplicado a los tres actores educativos y que además recoge datos sociodemográficos de los encuestados. En total la escala cuenta con 50 ítems, y todos ellos se responden bajo el formato de escala Likert (siempre, frecuentemente, rara vez, nunca, desconoce). 


\section{Tabla 2. Dimensiones que se deben evaluar}

1. Coordinación pedagógica entre educadora y asistente: grado en que la educadora y la asistente coordinan su accionar pedagógico, que va desde una coordinación administrativa-asistencial hasta una coordinación para el aprendizaje.

2. Gestión de recursos y espacios: grado de adecuación del espacio físico en que se desarrolla el taller y la propuesta de actividades TCC.

3. Gestión de recursos y materiales: gestionar el cuidado de los materiales reutilizables y reponer los fungibles.

4. Preparación de recursos materiales y mobiliario: la implementación de las actividades demanda la preparación anticipada de los materiales y el mobiliario necesario para realizar las experiencias científicas.

5. Criterios de selección de niños y niñas del taller: la conformación de grupos heterogéneos genera oportunidades de aprendizaje entre pares.

6. Explicación del fenómeno: la transposición didáctica es la competencia pedagógica que permite a las educadoras explicar fenómenos científicos a los niños y las niñas sin pérdida de riqueza conceptual.

7. Desarrollo de la competencia: movilización de conocimientos conceptuales, procedimentales y actitudinales en el contexto de actividades científicas elaboradas por competencias.

8. Retroalimentación y preguntas: la retroalimentación oral y gestual entrega valiosa información sobre el desempeño alcanzado, las adecuaciones que deben realizar y las estrategias por mejorar.

9. Participación de los niños y las niñas: los niños y las niñas son protagonistas del proceso de aprendizaje, con oportunidades de responder preguntas, experimentar, compartir resultados y observaciones.

10. Planificación de la actividad por parte de la educadora y la asistente: la planificación conjunta de la educadora y la asistente implica compartir modelos mentales sobre la actividad.

Una vez aplicado el instrumento se utilizó una prueba de fiabilidad conocida como alfa de Cronbach (Abad, Garrido, Olea y Ponsoda, 2006). El resultado indica que el instrumento tiene una buena validez interna (o,899). De todas maneras, al eliminar cuatro preguntas - que son aquellas que en menor medida se correlacionan con el resto de la escala-, los niveles de validez interna del instrumento aumentan a 0,914. A raíz de esto, para futuras mediciones, el instrumento ha sido corregido para lograr mejores niveles de fiabilidad.

La construcción del instrumento se ha desarrollado a partir de la importancia que tiene la relación que establecen el director, la asistente y la educadora de párvulos para la implementación exitosa de los talleres TCC. La influencia del director en los procesos de aprendizaje de los alumnos ha sido estimada en un $25 \%$, de acuerdo con investigaciones realizadas por Leithwood et al. (2004, citado en CEPPE, 2009).

En función del antecedente anterior se han establecido criterios para la ponderación de los puntajes de las diez dimensiones medidas. En la tabla 3 se muestran las ponderaciones asociadas a cada uno de los participantes de la investigación: 
Tabla 3. Ponderación de puntajes por actores

\begin{tabular}{|l|c|c|c|c|}
\hline \multicolumn{1}{|c|}{ Subindices } & Director & Educadora & Asistente & Total \\
\hline Relación entre la educadora y la asistente & 20 & 40 & 40 & 100 \\
\hline Gestión de recursos (espacios) & 50 & 20 & 30 & 100 \\
\hline Gestión de recursos (materiales) & 50 & 20 & 30 & 100 \\
\hline Preparación de recursos materiales y mobiliario para actividad & 20 & 30 & 50 & 100 \\
\hline Criterios para la selección de los niños(as) & 30 & 40 & 30 & 100 \\
\hline Explicación del fenómeno & 20 & 50 & 30 & 100 \\
\hline Desarrollo de la competencia & 20 & 50 & 30 & 100 \\
\hline Retroalimentación y preguntas & 20 & 50 & 30 & 100 \\
\hline Participación de los niños(as) & 20 & 50 & 30 & 100 \\
\hline Apropiación de la actividad por parte de la educadora y la asistente & 20 & 50 & 30 & 100 \\
\hline Total & 27 & 40 & 33 & 100 \\
\hline
\end{tabular}

De esta manera se ha construido el puntaje para cada establecimiento en función de las respuestas de los tres actores involucrados. Es por esta razón que han sido excluidos todos aquellos jardines en los cuales ha faltado la respuesta de uno de los implicados.
Además de los criterios recién mencionados se construyó un instrumento que evidenciara el nivel de apropiación de las competencias científicas de cada niño y niña. El instrumento fue un cuadernillo cuyo objetivo era evaluar las dimensiones descritas en la tabla 4:

\section{Tabla 4. Dimensiones del cuadernillo}

1. Disposición positiva hacia el aprendizaje: agrado verbal y gestual por las experiencias científicas.

2. Recuerdo de la experiencia: el nivel de precisión en la evocación del experimento, materiales utilizados y problema asociado.

3. Desarrollo de la competencia: articulación entre el conocimiento conceptual, procedimental y actitudinal en situaciones experimentales.

4. Trabajo en grupo: evidencias de intercambio de opiniones.

\section{Procedimiento}

El análisis de los datos se realizó con el software SPSS, versión 15.0, para lo cual se creó una base de datos que fue ingresada al programa. Posteriormente se realizaron análisis univariados y bivariados utilizando la estadística descriptiva e inferencial. Debido a las características de la muestra y a la selección de los participantes — que han sido principalmente to- dos los establecimientos que participaron en la implementación- y dado el tamaño de la muestra, no es posible asegurar la distribución normal de esta. Por dicho motivo se han empleado las pruebas correspondientes a la estadística no paramétrica (Siegel y Castellán, 2012) para medir los niveles de asociación entre la escala, las variables de clasificación (como sexo o edad) y las de estudio. 


\section{Resultados}

A continuación se presentan los resultados de las encuestas realizadas a los diferentes agentes educativos, a los estudiantes y sus relaciones (tabla 5).

\section{Tabla 5. Resultados generales de los} establecimientos

\begin{tabular}{|l|c|}
\hline \multicolumn{1}{|c|}{ Subindices } & Total (\%) \\
\hline $\begin{array}{l}\text { Coordinación pedagógica entre la educa- } \\
\text { dora y la asistente }\end{array}$ & 95,85 \\
\hline Gestión de recursos (espacios) & 76,8 \\
\hline Gestión de recursos (materiales) & 74,8 \\
\hline $\begin{array}{l}\text { Preparación de recursos materiales y mo- } \\
\text { biliario para actividad }\end{array}$ & 63,1 \\
\hline Criterios para la selección de los niños(as) & 54,1 \\
\hline Explicación del fenómeno & 83,9 \\
\hline Desarrollo de la competencia & 82,1 \\
\hline Retroalimentación y preguntas & 84,9 \\
\hline Participación de los niños(as) & 74,9 \\
\hline $\begin{array}{l}\text { Apropiación de la actividad por parte de la } \\
\text { educadora y la asistente }\end{array}$ & 74,6 \\
\hline
\end{tabular}

En la tabla 5 se observa que la dimensión más elevada es la coordinación pedagógica entre la educadora y la asistente, con un 90,85\%. El porcentaje más bajo es la dimensión criterios de selección de los niños(as), por lo que en general los establecimientos tuvieron ciertas dificultades para formar un grupo heterogéneo. También se encuentra disminuida la dimensión preparación de recursos materiales y mobiliario para actividad, lo que implica que en los establecimientos se hace difícil la preparación anticipada de los materiales y el mobiliario necesario para implementar las experiencias científicas.

En la tabla 6 se observa que el porcentaje más bajo es el de criterios para la selección de los niños(as). La preparación de recursos también se ve disminuida. Solo la coordinación pedagógica entre la asistente y la educadora y la gestión de recursos (materiales) se encuentran sobre un $80 \%$, lo que evidencia buenas prácticas en estos ámbitos. Las demás dimensiones están en el rango del $70 \%$, lo que indica que deberían ser mejoradas para las siguientes implementaciones.
Tabla 6. Resultados de los directores

\begin{tabular}{|l|c|}
\hline \multicolumn{1}{|c|}{ Subindices } & Total (\%) \\
\hline $\begin{array}{l}\text { Coordinación pedagógica entre la educa- } \\
\text { dora y la asistente }\end{array}$ & 88,0 \\
\hline Gestión de recursos (espacios) & 74,1 \\
\hline Gestión de recursos (materiales) & 80,4 \\
\hline $\begin{array}{l}\text { Preparación de recursos materiales y } \\
\text { mobiliario para actividad }\end{array}$ & 68,8 \\
\hline Criterios para la selección de los niños(as) & 52,3 \\
\hline Explicación del fenómeno & 72,1 \\
\hline Desarrollo de la competencia & 78,2 \\
\hline Retroalimentación y preguntas & 76,8 \\
\hline Participación de los niños(as) & 74,5 \\
\hline $\begin{array}{l}\text { Apropiación de la actividad por parte de } \\
\text { la educadora y la asistente }\end{array}$ & 70,3 \\
\hline
\end{tabular}

Tabla 7. Resultados de las educadoras de párvulos encuestadas por dimensión

\begin{tabular}{|l|c|}
\hline \multicolumn{1}{|c|}{ Subindices } & Total (\%) \\
\hline $\begin{array}{l}\text { Coordinación pedagógica entre la educa- } \\
\text { dora y la asistente }\end{array}$ & 87 \\
\hline Gestión de recursos (espacios) & 74,7 \\
\hline Gestión de recursos (materiales) & 75,1 \\
\hline $\begin{array}{l}\text { Preparación de recursos materiales y } \\
\text { mobiliario para actividad }\end{array}$ & 70,9 \\
\hline Criterios para la selección de los niños(as) & 57,8 \\
\hline Explicación del fenómeno & 82,7 \\
\hline Desarrollo de la competencia & 84,1 \\
\hline Retroalimentación y preguntas & 85,6 \\
\hline Participación de los niños(as) & 82,5 \\
\hline $\begin{array}{l}\text { Apropiación de la actividad por parte de la } \\
\text { educadora y la asistente }\end{array}$ & 72 \\
\hline
\end{tabular}

Se observa que el porcentaje más bajo es el de criterios para la selección de los niños(as), lo que significa que tuvieron dificultades para conformar grupos heterogéneos. También llama la atención la diferencia entre los porcentajes de coordinación y apropiación, por lo que se puede conjeturar que la coordinación puede ser comprendida como distribución de tareas, lo que no implica que se apropien de la actividad, es decir, que no compartan modelos mentales sobre la actividad (tabla 7). 
Tabla 8. Resultados de las asistentes de párvulos encuestadas por dimensión

\begin{tabular}{|l|c|}
\hline \multicolumn{1}{|c|}{ Subindices } & Total (\%) \\
\hline $\begin{array}{l}\text { Coordinación pedagógica entre la educa- } \\
\text { dora y la asistente }\end{array}$ & 92,6 \\
\hline Gestión de recursos (espacios) & 75,1 \\
\hline Gestión de recursos (materiales) & 73,8 \\
\hline $\begin{array}{l}\text { Preparación de recursos materiales y mobi- } \\
\text { liario para actividad }\end{array}$ & 72,9 \\
\hline Criterios para la selección de los niños(as) & 54,5 \\
\hline Explicación del fenómeno & 88 \\
\hline Desarrollo de la competencia & 89,8 \\
\hline Retroalimentación y preguntas & 87 \\
\hline Participación de los niños(as) & 85,2 \\
\hline $\begin{array}{l}\text { Apropiación de la actividad por parte de la } \\
\text { educadora y la asistente }\end{array}$ & 81,3 \\
\hline
\end{tabular}

Al igual que los resultados arrojados en las encuestas de los otros actores, la dimensión más baja es la de criterios para la selección de los niños(as). Además, la diferencia entre coordinación y apropiación de la actividad es porcentualmente importante, coincidiendo en este aspecto con los resultados de las encuestas a las educadoras (tabla 8).

Figura 2. Resultados de niños(as) encuestados

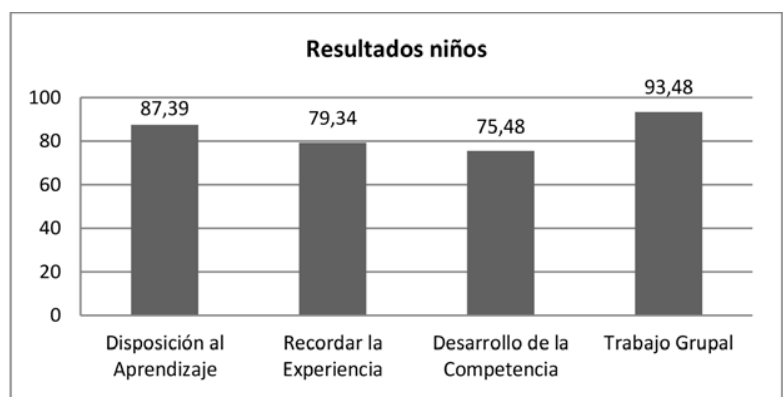

En la figura 2 se observa que el puntaje más alto se encuentra en trabajo grupal (93,48\%), lo que indica que los niños y las niñas trabajaron en grupo de manera efectiva. Le sigue la disposición al aprendizaje, con un $87,39 \%$, lo que indica que los niños y las niñas presentan una alta disposición al aprendizaje. Así mismo lograron recordar las experiencias cientificas en un $79,34 \%$ y la competencia fue desarrollada en un $75,48 \%$.

\section{Figura 3. Resultados de niños(as)/ Resultados de actores educativos}

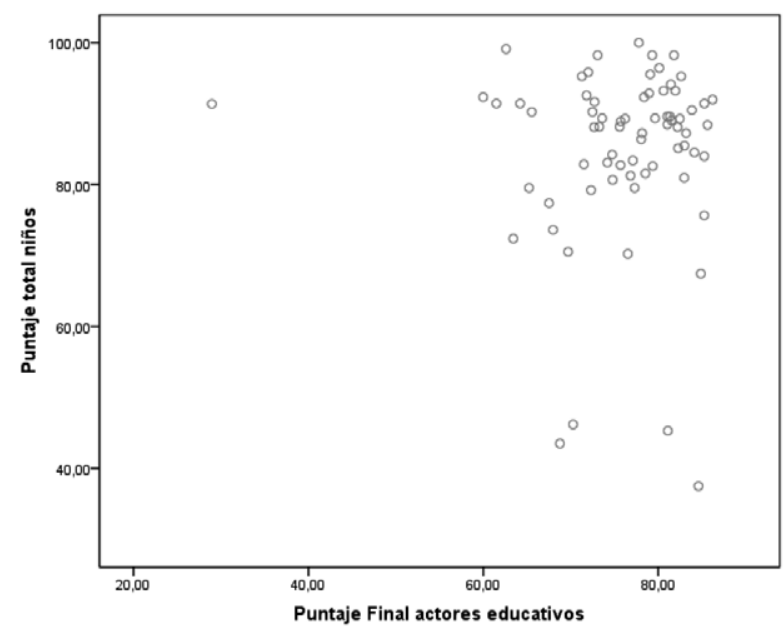

A partir del análisis de los resultados obtenidos por los actores educativos y por los niños y las niñas que participaron en la implementación de los talleres TCC, la figura 3 muestra la distribución de ambas variables.

En los resultados a nivel de establecimiento y de actor (tablas 5, 6, 7 y 8) se observó un alto porcentaje respecto a la dimensión coordinación pedagógica entre la educadora y la asistente, lo cual es consistente con la bibliografía existente, que ha puesto sobre el tapete la importancia de la colaboración. No obstante, este resultado también se relaciona con el rol que cumplen las asistentes de párvulos en la enseñanza. Nuestros supuestos eran que debería existir una coordinación pedagógica entre la educadora y la asistente para el buen desarrollo de la experiencia científica. Pues bien, para que esta coordinación se produzca es generalmente la educadora de párvulos quien puede otorgar ese espacio y empoderar a la asistente en su rol instruccional. Lo anterior guarda relación con la bibliografía que postula que actualmente las asistentes de párvulos ocupan gran cantidad de su tiempo en la instrucción de los ni- 
ños y las niñas (Barnes y Quimby, 1986; Wisconsin Department of Education, 1998; Gerber et al., 2001, citados en Ratcliff, Jones, Russell, Sheen y Hunt, 2011). Mientras que los autores Wirt et al. (2004), Hoffman y Sable (2006), Hampden-Thomson et al. (2007), citados en Ratcliff et al. (2011) sugieren que la mayoría del conocimiento sobre enseñanza de las asistentes ha sido adquirido mediante la observación de la educadora con la cual trabaja o de la planificación instruccional.

Todo esto nos demuestra que uno de los factores principales para la buena implementación de un programa educativo es la conformación de una dupla pedagógica que asigne roles y planifique en conjunto. Lo anterior es corroborado por los actores educativos por separado, quienes asignaron la mayor importancia a esta dimensión. Por otra parte, una de las dimensiones que se vio más disminuida, tanto a nivel de establecimiento como de actores educativos, fue la de los criterios de selección para los niños(as). Esto nos lleva a la discusión y cuestionamiento sobre qué es lo más óptimo para la implementación de este proyecto. Uno de los principios del proyecto piloto TCC-EP impuesto por ExploraConicyt exigía que la conformación de los talleres fuese heterogénea en relación con las edades de los niños y las niñas, fluctuando entre los 3 y 6 años. No obstante, en la práctica este tema generó muchos conflictos en las educadoras, primero, por problemas logísticos, como por ejemplo el que al conformar un grupo de niños heterogéneo, en ocasiones no tenían en dónde realizar las experiencias. Segundo, y lo más importante, es que a las educadoras se les hacía muy dificil trabajar con niños y niñas de diversas edades, pues no tenían las competencias pedagógicas para enfrentarse a un grupo heterogéneo, en donde en teoría cada individuo puede aportar y nutrir cada experiencia vivida a pesar de su edad.

En cuanto a la gestión de recursos (espacios), la gestión de recursos (materiales) y la preparación de los recursos materiales y mobiliario se observa que los tres actores educativos coinciden en evaluar estas dimensiones en el rango entre 80 y $70 \%$, a excepción de los directores, quienes evalúan la dimensión preparación de los recursos materiales y mobiliario como más baja que las anteriores (68,8\%). La implementación de las actividades demanda la preparación anticipada de los materiales y el mobiliario necesario para realizar las experiencias científicas. Esta tarea implica, generalmente, una coordinación entre los tres agentes educativos. Sería interesante indagar por qué el director percibe esta dimensión más baja que sus subalternas.

Las demás dimensiones que se relacionan con la práctica en el aula, en general, son percibidas con porcentajes altos por los tres actores educativos. No obstante, el director percibe estas dimensiones un poco más bajas que sus subalternas, quizás porque este se encuentra menos involucrado con lo que pasa en el aula y se preocupa más por la provisión de recursos, tiempos y espacios para la implementación de TCC.

Finalmente, en cuanto a los niños y las niñas, la dimensión más alta fue la de trabajo grupal, lo cual es muy importante dado que esta competencia es transversal y muy valiosa para toda la vida de un sujeto. Por otra parte, la dimensión más baja fue la de desarrollo de la competencia. Creemos que esto resulta natural dado que, en primer lugar, es un proyecto piloto; en segundo lugar, las competencias de las educadoras de párvulos y de las asistentes para enseñar ciencias pueden requerir de más instancias de formación y, en tercer lugar, el desarrollo de competencias es un proceso cíclico, que se espera que se desarrolle a lo largo del ciclo formativo de los niños y las niñas, es decir, no es necesario que los párvulos adquieran las competencias en esta etapa, pues tendrán más oportunidades para desarrollarlas posteriormente, estando en vías de desarrollo.

Finalmente, teniendo como base los resultados de la figura 3, se observa una tendencia positiva entre el grado de coordinación de los diferentes agentes educativos (director, educadora y asistente de párvu- 
los) y el resultado de los párvulos. En otras palabras, la calidad de la implementación de los talleres TCC por los agentes educativos marca una tendencia en las oportunidades de aprendizaje de los niños y las niñas que participan de este proyecto.

\section{Conclusiones}

Iniciamos este artículo con el propósito de establecer cómo las relaciones entre los actores educativos podían ser potencialmente un factor preponderante en la promoción y el desarrollo de competencias científicas en estudiantes entre 4 y 6 años. Los resultados nos permiten establecer las siguientes ideas clave:

- Tendencia positiva. En particular se observa una tendencia positiva entre el grado de coordinación de los agentes educativos con los resultados de las encuesta de los niños y las niñas. Esto podría ser interpretado como un buen argumento para promover el trabajo colaborativo entre los diferentes actores educativos en virtud de lograr aprendizajes de calidad en los estudiantes $y$, visto de una manera externa, para seguir promoviendo la implementación del programa Tus Competencias en Ciencias a lo largo del país.

- Colaboración/Coordinación. Los esfuerzos de colaboración se expresan en acciones prácticas, en este caso la realización de reuniones de coordinación entre educadora y asistente, con el objetivo de planificar la clase y acordar roles y distribución de tareas. Comprendemos que la coordinación no es un sinónimo de colaboración, pero contiene un componente práctico que permite que ambos actores dialoguen y desarrollen posturas compartidas frente a determinadas situaciones.

- Modelo de análisis de los resultados de las encuestas. La ponderación por actor educativo de cada una de las categorías desarrolladas es una apuesta interesante ya que permite, a partir de la bibliografía, determinar el grado de incidencia que el actor tiene en relación con ella. Esta determinación, que podría parecer arbitraria, está lejos de serlo, ya que se encuentra apoyada en estudios que indican que el primer factor de incidencia en la calidad de los aprendizajes es el docente, y el segundo es el director. Este antecedente, asociado a las atribuciones propias de cada cargo, sustenta las ponderaciones de cada uno de los actores.

Sin embargo, una mayor coordinación entre los actores educativos demanda al director gestionar el tiempo de los docentes para que ellos realicen reuniones en donde puedan analizar y planificar cada actividad en profundidad, asignando roles y revisando los experimentos y materiales. Bajo el supuesto de que el tiempo escolar siempre es escaso, priorizar este tipo de actividades da a entender que en esa cultura escolar se valora el trabajo conjunto. Esta consideración no es producto del azar, es la conjunción de creencias individuales y colectivas que conforman la cultura de ese grupo humano, que ha desarrollado redes sociales sobre las que se apoya el capital intelectual que nutre el trabajo profesional.

La ausencia de estas condiciones puede derivar en que la implementación de iniciativas de programas educativos para promover competencias (por ejemplo, TCC-EP) sea exitosa solo en sus inicios, ya que estas se apoyan fundamentalmente en el entusiasmo e interés de las docentes protagonistas. De ocurrir esto, pasado un tiempo termina por decaer el ánimo y la iniciativa paulatinamente se olvida y se deja de implementar en aquellos que mayor impacto y frutos genera: nuestros estudiantes.

\section{Agradecimientos}

Este artículo hace parte del Proyecto Fondecyt 11100402 (2010-2012) "Creencias sobre ciencia, su enseñanza y aprendizaje en educadores de párvulos y su influencia en la implementación del programa 
TCC" (abrev.) Producto científico patrocinado y financiado por el Fondo Nacional de Desarrollo Científico y Tecnológico (Fondecyt-Conicyt), Chile.
Vicerrectoría de Investigación y Estudios Avanzados. Pontificia Universidad Católica de Valparaíso, Chile. http:// www.vriea.ucv.cl/

\section{Referencias}

Abad, F., Garrido, J., Olea, J. y Ponsoda, V. (2006). Introducción a la psicometría: Teoría clásica de los test y teoría de respuesta al item. Madrid: Universidad Autónoma de Madrid.

Arrowsmith, T. (2004). Distributed leadership: three questions, two answers. A review of the Hay Group Education research. Management in Education, 18, 30-33.

Asún, R. (2006). Construcción de cuestionarios y escalas: el proceso de la producción de información cuantitativa. En Canales, M. Metodología de Investigación Social (pp. 63-113). Santiago: Lom.

Ávila, J. (2008). Department networks and distributed leadership in schools. School Leadership \& Management, $28(2), 159-187$.

Bennett, N., Wise, C., Woods, P. y Harvey, J. (2003). Distributed Leadership. Full Report. Gloucestershire: National College for School Leadership.

Bogler, R. (2001). The influence of Leadership Style on Teacher Job Satisfaction. Educational Administration Quarterly, 37 (5), 662-683.

Bolden, R. (2007). Distributed Leadership. En Marturano, A. y Gosling, J. (eds.), Leadership, The Key Concepts (pp. 99-110). Abingdon: Routledge.

Bowman, B., Donovan, M. S. y Burns, M. S. (2001). Eager to learn: Educating our Preschoolers. Washington DC: National Academy Press.

Bryk, C., Camburn, E.y Seashore, K. (1999). Professional Community in Chicago Elementary Schools: Facilitating Factors and Organizational Consequences. Educational Administration Quarterly, 35 (suplemento de diciembre), 751-781.

Burke, C., Stagl, K., Salas, E., Pierce, L.y Kendall, D. (2006). Understanding Team Adaptation. A Conceptual Analysis and Model. Journal of Applied Psychology, 91 (6), 1189-1207.

Bush, T. (2008). From Management to Leadership. Semantic or Meaningful Change? Educational Management Administration \& Leadership, 36 (2), 271-287.

CEPPE (2009). Prácticas de liderazgo directivo y resultado de aprendizaje: hacia conceptos capaces de guiar la investigación empírica. Revista Iberoamericana sobre Calidad, Eficacia y Cambio en Educación, 7 (3), 18-33.

Conley, S., Fauske, J. y Pounder, D. (2004). Teacher Work Group Effectiveness. Educational Administration Quarterly, 40 (5), 663-703. 
Cranston, J. (2009). Holding the Reins of the Professional Learning Community: Eight Themes from Research on Principals' Perceptions of Professional Learning Communities. Canadian Journal of Educational Administration and Policy (CJEAP), 9o. Recuperado el 12 de febrero de 2013 de: http://www.umanitoba.ca/ publications/cjeap/articles/cranston.html

Dinham, S., Aubusson, P.y Brady, L. (2008). Distributed Leadership as a Factor in and Outcome of Teacher Action Learning. International Electronic Journal For Leadership in Learning, 12 (4), s/n.

Dwyer, M. C., Chait, R. y McKee, P. (2000). Building strong foundations for early learning: The U.S. Department of Education's guide to high-quality early childhood education programs. Washington, DC: U.S. Department of Education, Planning and Evaluation Service.

Espinosa, L. M. (2002). High quality preschool:Why we need it and what it looks like [Electronic version]. Preschool Policy Matters, 1, 1-10. Recuperado 27 de enero de 2013 de: http://nieer.org/resources/policybriefs/1.pdf.

García Huidobro, J. (2006). Formación inicial de educadoras(es) de párvulos en Chile. En Foco. Corporación Expansiva. Recuperado 18 de febrero de 2013 de: http://www2.expansiva.cl/media/en_foco/documentos/19062006105036.pdf.

González-Weil, C., Martínez, M., Martínez, C., Cuevas, K. y Muñoz, L. (2009). La educación científica como apoyo a la movilidad social: desafíos en torno al rol del profesor secundario en la implementación de la indagación científica como enfoque pedagógico. Estudios pedagógicos (Valdivia), 35 (1), 63-78.

Gronn, P. (2002). Distributed Leadership. Oxford: Oxford University Press. En Bennett, N. et al. (2003). Distributed Leadership. Full Report. Gloucestershire: National College for School Leadership.

Gropen, J., Clark-Chiarelli, N., Ehrlich, S.y Thieu, Y. (2011). Examining the Efficacy of Foundations of Science Literacy: Exploring Contextual Factors. Evanston, IL: Society for Research on Educational Effectiveness.

Halverson, R., Prichett, R., Grigg, J. y Thomas, C. (2007). The New Instructional Leadership: Creating Data-Driven Instructional Systems. Schools. Journal of School Leadership, 17 (2), 159-194.

Hargreaves, D. (2001). A Capital Theory of School Effectiveness and Improvement. British Educational Research Journal, 27 (4), 487-503.

Harris, A. (2002). Distributed Leadership in Schools: Leading or Misleading? En British Educational Leadership Management \& Administration Society Conference. Birmingham. ED 496881.

Harris, A. y Townsend, A. (2007). Developing leaders for tomorrow: releasing system potential. School Leadership \& Management, 27 (2), 167-177.

Harris, A.y Spillane, J. (2008). Distributed leadership through the looking glass. Management in Education, 22 (1), 31-34.

Helburn, S. W.y Bergmann, B. R. (2002). America's child care problem: The way out. New York: Palgrave.

Hernández, R., Fernández, C.y Baptista, M. (2010). Metodología de la investigación. México: McGraw-Hill. 
Izquierdo, M. y Adúriz-Bravo, A. (2003). Epistemological foundations of school science. Science \& Education, 12 (1), 27-43.

Michaels, S., Shouse, A. y Schweingruber, H. (2007). Ready, set, science!: Putting research to work in K-8 science classrooms. New York: National Academics Press.

Ogawa, R. y Bossert, S. (1995). Leadership as an organizational quality. En Ávila, J. (2008). Department networks and distributed leadership in schools. School Leadership \& Management, 28 (2), 159-187.

Quiroga, M., Cafena, D., Merino, C. y Olivares, C. (2012). Ciencia, competencias y enseñanza de las ciencias en niños de 2 a 6 años. En Badillo, E., García, L., Marbà, A. y Briceño, M. (coords.). El desarrollo de competencias en la clase de ciencias y matemáticas (pp. 371-397). Mérida: Ediciones Universidad de los Andes.

Ratcliff, N., Jones, C., Russell, S., Sheen, H. y Hunt, G. (2011). Paraprofessionals in early childhood classrooms: an examination of duties and expectations. Early Years: An International Journal of Research and Development, 32 (2), 163-179.

Rebollo, M. (2010). Análisis del concepto de competencia científica: definición y sus dimensiones. En / Congreso de Inspección de Andalucía: competencias básicas y modelos de intervención en el aula. Andalucía: Consejería de Educación.

Riveros-Barrera, A. (2012). La distribución del liderazgo como estrategia de mejoramiento institucional. Educ. Educ. 15 (2), 289-301.

Siegel, S.y Castellán, N. J. (2012). Estadística no paramétrica: aplicada a las ciencias de la conducta. México: Trillas.

Southard, M., Muldoon, J., Porter, P.y Hood, D. (1997). School Councils' Perceptions of Site-Based Decision Making: Challenges and Benefits. En Annual Meeting of the American Educational Research Association. Chicago, IL. ED 461149 .

Spillane, J., Halverson, R. y Diamond, J. (2001). Investigating school leadership practice: A distributed perspective. En Ávila, J. (2008). Department networks and distributed leadership in schools. School Leadership \& Management, 28 (2), 159-187.

Tyler, W. (1996). Organización escolar. Madrid: Morata.

Woods, A., Bennett, N., Harvey, J. y Wise, C. (2003). Variabilities and Dualities in Distributed Leadership: Findings from a systematic Literature Review. En Arrowsmith, T. (2004). Distributed leadership: three questions, two answers. A review of the Hay Group Education Research. Management in Education, 18, 30-33. 